In:

Reinhard Johler, Ansgar Thiel, Josef Schmid, Rainer Treptow (Hg.):

Europa und seine Fremden.

Die Gestaltung kultureller Vielfalt als Herausforderung.

Bielefeld, transcript, 2007

\section{Zum Paradigma kultureller Differenz}

\section{MARTIN SÖKEFELD}

\section{Einleitung}

In diesem Beitrag diskutiere ich einige Aspekte des Verhältnisses von Kultur und Migration in Deutschland. Ich will gleich die Bemerkung vorausschicken, dass ich die Rolle, die „Kultur“ als Konzept in der deutschen Migrationsdebatte explizit oder implizit spielt, für höchst problematisch erachte.

Vordergründig ist „Kultur" ein strittiges Konzept in der Debatte, wie unschwer an den beiden umstrittenen Begriffen der „Leitkultur" und der „Multikultur" zu erkennen ist. Diese beiden Begriffe bestimmen anscheinend einander entgegengesetzte Positionen. Tatsächlich liegt ihnen jedoch trotz des scheinbaren Gegensatzes ein ähnliches Verständnis von Kultur zugrunde. Für beide Begriffe bezeichnet Kultur in erster Linie Differenz

Es ist eine der wichtigsten Aufgaben der Kultur- und Sozialwissenschaften, über die Begriffe zu reflektieren, die wir benutzen, um die soziale Welt zu beschreiben und zu deuten. Die Beschreibung und Deutung ist nie nur passiv, sondern sie hat konkrete soziale Auswirkungen. Wenn die soziale Welt, wie Max Weber sagte, eine sinnhaft konstituierte Welt ist, dann hat der Sinn; den wir dieser Welt geben - etwa dadurch, dass wir bestimmte Begriffe mit einem bestimmten Verständnis verwenden, um eine Situation zu erfassen - Folgen für die soziale Welt. Dies gilt auch für wissenschaftliche Begriffe, die keinesfalls außerhalb des gesellschaftlichen Zusammenhangs stehen. Anthony Giddens prägte den Begriff der „doppelten Hermeneutik“ für das wechselseitige Verhältnis von wissenschaftlicher Begrifflichkeit und dem Alltagsverständnis von Konzepten. Beide Diskursbereiche verhalten sich reziprok zueinander und wirken aufeinander ein. Giddens schreibt: 
[...] die Aneignung der von den Sozialwissenschaftlern erfundenen Fachbegriffe und "[...] dien [durch Laien] [...] kann diese zu konstituierenden Elementen des ,GegenstanTheorien [durch Laien] [...] kann diese zu konstituierenden Elementen des , Gegenstan-
des" machen, für dessen Charakterisierung sie geprägt wurden, und aus diesem Grund ihren Verwendungskontext verändern" (1984: 95).

Besonders Michel Foucault hat die konstituierende Macht von Diskursen betont, die unter anderem darin besteht, festzulegen was denk- und sagbar ist und was nicht. Diese konstituierende Macht gilt natürlich auch für Begriffe als zentrale Elemente der Diskurse.

Die Wirkung von Diskursen auf die Migrationspolitik in Deutschland ist ziemlich offensichtlich und wurde vielfach untersucht. Anhand etwa der Abfolge der Begriffe, die der Migrationsdiskurs verwendet hat, um die Menschen, die da gekommen und geblieben sind zu bezeichnen, kann man etwa sehr gut die Umorientierungen der Migrationspolitik nachvollziehen: Von den Gastarbeitern der sechziger und siebziger Jahre, die als nur temporär Anwesende betrachtet wurden, mit der Folge, dass die Migrationspolitik damals einzig auf ihre Rückkehr ausgerichtet war, über die Ausländer der achtziger und frühen neunziger Jahre, die zwar geblieben waren, aber keineswegs dazugehörten, und deren politische und gesellschaftliche Partizipation daher kaum gefördert wurde, bis zum aktuellen Begriff der Zuwanderer, der zwar einerseits eher widerwillig das Bleiben der "Wanderer" anerkennt und teils sogar die Notwendigkeit weiterer Zuwanderung "akzeptiert, gleichzeitig aber stets die mangelnde „Integration“ der Zugewanderten betont und somit die nachdrückliche Reserviertheit gegenüber Migranten, von der die bundesdeutsche Debatte geprägt ist, keineswegs aufgegeben hat. Ich denke, es ist kein Zufall, dass sich nach der Einsicht, dass allen gegenteiligen Bekundungen der Vergangenheit zum Trotz Deutschland eben doch eine Einwanderungsgesellschaft ist, keineswegs der naheliegende Begriff „Einwanderer" als Bezeichnung für Migranten in Deutschland durchgesetzt hat. Der Griff zum anderen Präfix lässt sich durchaus als Beharren auf einem gewissen Ressentiment interpretieren.

\section{Migranten als Problem}

Die durchgängige Konstante der deutschen Migrationsdebatte ist, dass Migration, oder schlicht und einfach „die Migranten", als Problem betrachtet werden. Über die Konstante der Problematisierung kann auch nicht hinwegtäuschen, dass die "Gastarbeiter" ursprünglich geholt wurden, um ein Problem zu lösen, nämlich den Arbeitskräftemangel im Wirtschaftswunderland, und dass „Zuwanderer" heute als Lösung eines anderen Problems diskutiert werden, des Problems der demographischen Schieflage der Gesellschaft.

Die Problematisierung der Einwanderung wird in der öffentlichen Thematisierung derzeit häufig als neue Erkenntnis dargestellt, welche die bisherige
Wahrnehmung einer Revision unterziehe. So könnte man, wenn man die Debatte der letzten zwei oder drei Jahre verfolgt, zu dem Schluss kommen, dass das „Problem" mit den Einwanderern erst jetzt erkannt worden ist, nachdem man in den Jahrzehnten eines unbeschwerten Multikulturalismus die Augen davor verschlossen habe. Erst heute seien die „Integrationsdefizite“ und die „Parallelgesellschaft" der Einwanderer offenbar geworden. Diese Wahrnehmung ist jedoch eine Fiktion, denn weder hat es in Deutschland jemals eine generelle Politik des Multikulturalismus gegeben, noch ist die Thematisierung von Immigranten als „Problem“ etwas Neues. Schon 1973 widmete Der Spiegel den „Ausländern“ eine erste alarmistische Titelgeschichte. Der Titel lautete damals: "Ghettos in Deutschland - eine Million Türken in Deutschland.“ Im Heft begann der Artikel mit der Überschrift: „Die Türken kommen - rette sich, wer kann". ${ }^{\text {I Vom }}$ „Ghetto“ zur „Parallelgesellschaft“ - jenseits der Wortwahl hat sich die Wahrnehmung kaum geändert.

Die Kategorisierung von Migranten als Problem für die Gesellschaft ist in Deutschland seit langem zu einer sozialen Tatsache im Sinne Durkheims geworden, die weitgehend unhinterfragt eine Prämisse der gesellschaftlichen Wahrnehmung darstellt (vgl. Griese 2002: 34). Ich will damit weder sagen, dass es keine Probleme in Zusammenhang mit Migration gibt, noch dass Politik und Diskurse keine Veränderung erfahren haben. Eine wichtige Veränderung war zweifelsohne die Reform des Staatsangehörigkeitsrechts, die Anfang 2000 wirksam wurde, und die erstmals ein Element des ius soli in das deutsche Recht einbrachte. Aber die Geschichte dieser Reform zeigt wiederum die Konstante der Politik: In der Kampagne gegen die Reform, die die CDU zur Landtagswahl in Hessen 1999 startete, standen ,die Ausländer" als Problem für Deutschland im Zentrum, es ging um Abgrenzung, und viele Bürger in Hessen nutzten die Chance, „gegen Ausländer zu unterschreiben." Was als eine Reform der Öffnung gedacht war, geriet nur halbherzig, mit noch teils unabsehbaren rechtlichen Folgen.

Auch im Diskurs verschieben sich die Themen. Wurde in den siebziger und achtziger Jahren etwa die oft benachteiligte Situation von Immigrantinnen v.a. unter dem Stichwort patriarchalischer Familienstrukturen abgehandelt, so wird heute in erster Linie „der Islam“ für problematische Geschlechterverhältnisse verantwortlich gemacht. Andere Probleme sind hinzugekommen, die früher weniger beachtet wurden, wie die schulische Situation und die geringen Bildungschancen junger „Migranten“. Heute wird die Problemdebatte v.a. von der Angst vor Islamismus und Terrorismus dominiert. Als problematisch gelten somit heute in erster Linie muslimische Migranten.

Die Problematisierung von Migration ist nicht nur eine Konstante allgemeinöffentlicher und politischer Diskurse, sie charakterisiert ebenso weite Teile des wissenschaftlichen Diskurses über Migration in Deutschland. Dies gilt für verschiedene Disziplinen, wie die Pädagogik, die Sozialpsychologie oder die Sozio-

1 Der Spiegel, Nr. 31, 30. Juli 1973. 
logie. Die Pädagogik stellte Einwanderer als defizitäre Wesen dar, denen mit spezifischen Maßnahmen geholfen werden musste. Hamburger et al. schrieben schon 1984, dass die Maßnahmen der Ausländerpädagogik dazu beitrugen, „dass Situation und Status der Ausländer zunehmend jener der Behinderten, Sonderschüler, Obdachlosen usw. ähneln, nämlich der der abgegrenzten und handhabbaren Randgruppe, die Gegenstand von Programmen wissenschaftlicher wie praktischer Art ist" (Hamburger et. al. 1984: 33).

Diese Perspektive gilt nicht nur für die Erziehungswissenschaften. Auch die soziologische Ausländerforschung war stark ,problemorientiert" und nahm Einwanderer stets als ,soziales und politisches Problem wahr“ (Treibel 1988: 11). In ähnlicher Weise beschäftigte sich die Sozialpsychologie v.a. mit den Sozialisations- und Identitätsproblemen von Einwanderern, besonders von Jugendlichen (vgl. Gontovos 2000).

\section{Kultur als Problem}

Warum sind Gastarbeiter, Ausländer oder Zuwanderer dem Diskurs zufolge so inhärent problematisch? Ein Erklärungsmodell begann sich Ende der 1970er Jahre durchzusetzen: Kultur. Einwanderer sind danach deshalb so problematisch, weil sie einer anderen Kultur angehören als „wir", weil „ihre“ Kultur so anders ist als ,unsere“. In der Pädagogik setzte sich die „Differenzhypothese“ als Erklärungsmodell gegen die „Defizithypothese“ durch. Kultur wird dabei in ein Assimilations- oder Integrationsmodell eingeordnet: Danach verlangte die Integration in Deutschland einerseits von Einwanderern, dass sie ihre „Heimatkultur" wenigstens teilweise aufgeben und dafür Elemente der "deutschen Kultur" übernehmen. Andererseits werden dem Modell zufolge aber die Einwanderer durch ihre Herkunftskultur gerade daran gehindert, sich zu integrieren. Es kommt daher zum „Kulturkonflikt“‘. Die These des Kulturkonfliktes dominierte weite Bereiche des wissenschaftlichen Diskurses über Einwanderung, besonders in den 1980er Jahren. Inzwischen, seit Samuel Huntingtons „Clash of Civilizations", ist sie sogar als These zur Erklärung globaler Konflikte hoffähig geworden und es scheint, dass sie, v.a. in der Politik, Anhänger gewinnt.

Heute wird in der Migrationsdebatte nicht mehr so banal argumentiert wie in den siebziger Jahren, als etwa Schrader et al. in einer Studie über die „Zweite Generation“ schrieben: „Seine einmal übernommene kulturelle Rolle kann der einzelne nicht mehr abwerfen: Er ist Deutscher, Franzose, Türke oder Italiener" (Schrader et al. 1976: 58). Die Argumente sind heute subtiler. Dennoch wird auch die heutige Debatte von zwei Elementen eines höchst problematischen Kulturkonzeptes dominiert. Diese sind die Betonung von Grenzen und ein impliziter Determinismus. Die Betonung von Grenzen zeigt sich darin, dass auch der heutige Migrationsdiskurs fast durchgängig mit der Dichotomie von „wir" und „den anderen“ arbeitet. „Wir“, das sind „die Deutschen“, und die Anderen, das sind „die Migranten“. Die Ausgrenzung der Anderen dient dabei der Selbstvergewisserung, der Identität des Eigenen.

Auch die Betonung von „Integration“, dem dominanten Konzept des gegenwärtigen Diskurses, kann über die Zentralität der Dichotomie nicht hinwegtäuschen. Denn die Integrationsdebatte ist von der Betonung der Integrationsdefizite der Migranten beherrscht und zieht somit die Grenze, die sie ja eigentlich überwinden möchte, ständig neu. Man kann sogar zu dem Schluss kommen, dass gerade der Integrationsdiskurs mit seiner ständigen Thematisierung von Defiziten Integration, was immer genau darunter verstehen ist, dadurch verhindert, dass er die Kategorien, die vorgeblich aufgelöst werden sollen, permanent neu konstituiert. Die Dichotomie zwischen „Deutschen“ und „Migranten“ wird dadurch jedenfalls nicht verwischt, und es stellt sich die Frage, ob das überhaupt beabsichtigt ist. Darin zeigt sich der implizite Determinismus, der auf die kurze Formel gebracht werden kann: Einmal Migrant - immer Migrant. So ist heute bereits von der „,vierten Generation“ der Migranten die Rede, obwohl sich der Begriff, genau betrachtet, selbst ad absurdum führt. Sind doch diese „Migranten“ der vierten Generation bereits drei Generationen von denen entfernt, die tatsächlich einmal gewandert sind. Warum gelten die Angehörigen der „vierte Generation“ dann überhaupt noch als „Migranten"? Der Begriff hat nur dann Sinn, wenn man davon ausgeht, dass die Wanderung der ersten Generation quasi „vererbt" wird und ihre ursprüngliche Zugehörigkeit eine generationsübergreifende determinierende Kraft hat, dass sie Bedingungen setzt, denen auch die Kinder der Kindeskinder nicht entkommen können.

Dahinter steckt die Vorstellung, dass die Grenze zwischen „uns“ und „den Anderen“ tatsächlich nie aufgelöst werden kann. „Migranten“, egal welcher $\mathrm{Ge}$ nerationszugehörigkeit, werden als fortwährend „Fremde“ wahrgenommen und behandelt. Sie werden nie $\mathrm{zu}$ „Eigenen“. Dieses Verständnis ist nicht weit entfernt vom oben zitierten Satz von Schrader et al. (1976): Die kulturelle Rolle kann der einzelne auch über die Generationen hinweg nicht ablegen, er bleibt Deutscher oder etwa Türke.

Hinter dieser fortwährenden Kategorisierung der Einwanderer und ihrer Nachkommen als „Migranten“ steckt ein problematisches Konzept von Kultur, welches das Selbstverständnis der "Deutschen“ ebenso bestimmt wie ihr Verhältnis zu den „Anderen“. Es ist das alte, auf Herder zurückgehende Konzept von Kultur als dem, was ein "Volk" oder eine „Nation" definiert und sie von anderen unterscheidet. Herder pluralisierte das Kulturkonzept. Während vor Herder Kultur „oben" und „unten" in einer Geselischaft voneinander unterschied - die oben, der Adel, der Klerus, hatten Kultur, die unten, etwa die Bauern, nicht - setzte Herder die Idee von verschiedenen Kulturen in die Welt. Danach unterscheiden sich etwa Deutsche und Franzosen nicht mehr v.a. politisch - dadurch, dass sie in unterschiedliche Machtbereiche gehörten - sondern kulturell voneinander. Kultur wurde zum „Erbe“ eines Volkes, das sein Schicksal bestimmt und eine Art „natürlicher" Differenz zu anderen Völkern begründet. 
Die von Herder geprägte Idee einer „Kulturnation“ prägt bis heute das deutsche Selbstverständnis. Die Vorstellung von Kulturen im Plural als abgegrenzt und voneinander unterschieden ist den politisch anscheinend so verschiedenen Konzepten von Multikulturalismus und Leitkultur gemeinsam. Beide gehen von der Zentralität kultureller Unterschiede und von der Existenz differenter „Kulturen" als abgegrenzten Einheiten aus; sie unterscheiden sich in erster Linie in der Wertung dieser Differenz.

Meine eigene Disziplin, die Ethnologie, hat nicht unwesentlich zur Popularisierung dieser Vorstellung von Kultur beigetragen. In der Ethnologie war Franz Boas der Begründer eines pluralen Kulturkonzeptes. Seine Absicht war durchaus löblich, wollte er doch Kultur als Basis von Differenz an die Stelle der ethnozentrischen und hierarchisierenden Ideen von „Rassen“ und evolutionären Unterschieden setzen. Derrida hat darauf hingewiesen, dass die Auslöschung eines Begriffs oder einer Bedeutung und ihre Ersetzung durch einen anderen nie vollständig gelingt, sondern dass stets Spuren von dem, dessen man sich entledigen wollte, zurückbleiben. Das vorgeblich ausgelöschte Alte belegt das Neue mit seinem Bann. Dies gilt auch für das Verhältnis von „Rasse“ und Kultur. ${ }^{2}$

Das Kulturkonzept hat zwei problematische Aspekte des „Rasse“begriffs übernommen. Der erste Aspekt ist der Hang zum Determinismus. So wie die „Rassen"zugehörigkeit vermeintlich das Individuum auf eine bestimmte Weise determiniert, wird auch Kultur als etwas gedacht, dass das Individuum in gewissem Maße determiniert, etwa durch in der Sozialisation vermittelte Normen und Werte. Der zweite problematische Aspekt ist die Idee des Unterschieds selbst. Genauso wie es als möglich galt, „Rassen“ klar voneinander zu unterscheiden, wurden auch scharfe Grenzen zwischen Kulturen gezogen und darüber hinaus als kongruent mit geographischen, politischen oder sprachlichen Unterschieden postuliert. Das Kulturkonzept homogenisiert nach innen und heterogenisiert nach außen und überzeichnet damit Unterschiede. Wie die evolutionäre Stufenleiter oder das „Rassen“paradigma beinhaltet auch das Paradigma kultureller Differenz Hierarchie, Bewertung (meistens die Abwertung der „Anderen") und Dominanz. Die durch Kultur begründete Differenz zwischen Menschen ist nicht neutral oder unpolitisch.

Spätestens seit den 1980er Jahren wurde sich die Ethnologie jedoch mehr und mehr bewusst, dass diese Vorstellung weniger einem empirischen Befund entsprach als einer bestimmten Form der Repräsentation. ${ }^{3}$ Ethnologen erkannten, dass „Kultur" und „Kulturen" nicht nur der Gegenstand, sondern auch ein Artefakt der Ethnologie, bzw. anderer Diskurse, die mit diesen Konzepten arbeiteten, waren. Für die Produktion des „Anderen“ durch die ethnologische Perspektive

2 Stolcke (1995) hat überzeugend dargestellt, wie das Kulturkonzept auch im Diskurs der neuen Rechten weitgehend das "Rasse"konzept ersetzt und dieselbe ausgrenzende Rolle spielt, wie zuvor die „Rasse“.

3 Für einen Überblick dieser Debatte und eine kritische Reflexion siehe Fuchs/Berg 1993. wurde der Begriff des Othering geprägt. Dahinter steht die Erkenntnis, dass „die Anderen“, die die Ethnologie untersucht, nicht einfach ,an sich“ anders sind, sondern durch den ethnologischen Blick und durch die wissenschaftliche Repräsentation $\mathrm{zu}$ anderen gemacht, gewissermaßen „,verandert", verfremdet werden. Die Konsequenz dieser Erkenntnis war eine radikale Reflexion und Kritik ethnologischer Konzepte und Herangehensweisen, sowie, wenigstens teilweise, eine Neubestimmung des Gegenstandes der Ethnologie. In der Forschung geht es sei-

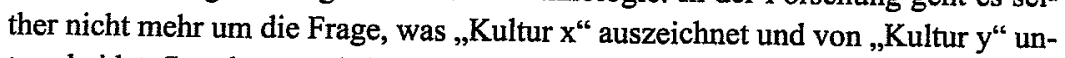
terscheidet. Stattdessen wird gefragt, wie gesellschaftliche Auseinandersetzungen Kulturen als Repräsentation hervorbringen, etwa dadurch, dass bestimmte Selbstund Fremdbilder entworfen werden, welche Akteure daran beteiligt sind, und wie diese Repräsentationen von Kulturen auf soziales Handeln zurückwirken und sich in gesellschaftliche Strukturen niederschlagen. ${ }^{4}$

Der zweite Schritt, die Untersuchung der gesellschaftlichen Wirkung und Folgen kultureller Repräsentationen, ist besonders wichtig, wird doch besonders konstruktivistischen und so genannten postmodernen Perspektiven häufig vorgeworfen, sie würden „Kulturen", und alle möglichen Formen von Gruppen als bloße Fiktionen, als diskursive Konstruktionen ohne Wirklichkeitsgehalt betrachten. Diskursive Konstruktionen können jedoch dadurch, dass sie geglaubt und für wirklich gehalten werden, für die gesellschaftlichen und politischen Verhältnisse entscheidende Konsequenzen haben und somit sehr real werden. Obwohl also die konstruktivistische Perspektive die Realität diskursiver Konstruktionen - im Sinne ihrer gesellschaftlichen und politischen Wirkmächtigkeit - nicht bestreitet, liefert sie die Möglichkeit zu ihrer Dekonstruktion, die genau darin besteht, aufzuzeigen, wie Kulturen, Differenzen, usw. gesellschaftlich produziert werden und sich in sozialen Strukturen niederschlagen. Sie stellt damit den Glauben an die Normalität oder Natürlichkeit kultureller Differenzen in Frage und entfaltet damit im besten Fall ihre eigene, aufklärende gesellschaftliche Wirkung.

\section{Hybridität statt Differenz}

Ein Konzept, dass in diesem Zusammenhang in der akademischen Debatte eine wichtige Rolle spielt, ist das der Hybridität. In gewisser Hinsicht ist Hybridität zu einem Gegenkonzept gegen die konventionelle Vorstellung abgegrenzter Kulturen und gegen die Objektivierung kultureller Differenz geworden. Das Konzept lenkt die Aufmerksamkeit auf Übergänge und Passagen, auf Zwischenräume, in denen sich das scheinbar Getrennte „vermischt“. Statt kultureller Differenzen und Grenzen steht „Kontinuität“ im Zentrum des Begriffs. Hybridität ist ein sehr komplexes, viel diskutiertes und viel kritisiertes Konzept, das ich in diesem Rahmen nicht annähernd erschöpfend ausleuchten kann. Dem Begriff wurde u.a.

4 Zur Diskussion über den Kulturbegriff siehe Sökefeld 2001. 
vorgeworfen, dass er letztlich das Paradigma kultureller Differenz bestätige, da Hybridität, etwa im Sinne der Genetik, die Vermischung von zwei zuvor getrennten Entitäten bezeichne. ${ }^{5}$ Darum geht es jedoch gerade nicht, sondern eher um eine Umkehrung der Perspektive. Hybridität bezeichnet dann die Vorstellung, dass Übergänge, Passagen und Zwischenräume genauso „normal“ sind wie Prozesse der Vermischung, dass es keine ursprüngliche „Reinheit“ von und Trennung zwischen Kulturen gibt, und dass Kulturen - wenn man denn das Konzept im Plural überhaupt beibehalten möchte - nicht als scharf voneinander abgegrenzt gedacht werden können, sondern als Gebilde, die an den Rändern „ausfransen“ und ohne scharfe Grenze in einander übergehen.

Eine solche Vorstellung von Kultur erscheint für den common sense heute ungewöhnlich und schwer nachvollziehbar, hat doch die dominante politische Institution der Moderne, der Nationalstaat, seit dem ausgehenden achtzehnten Jahrhundert erfolgreich alles daran gesetzt, die gegenteilige Idee durchzusetzen und zur Normalität zu machen. Der Nationalstaat ist auf scharfe, eindeutige Grenzen gebaut, auf die Eindeutigkeit der Grenze seines Territoriums genauso wie auf die Eindeutigkeit der Unterscheidung, wer ihm angehört und wer nicht. Hybridität und Ambivalenz der Zugehörigkeit hat der Nationalstaat aktiv bekämpft und als unzuverlässig, d.h. nicht eindeutig zugehörig geltende Bevölkerungsgruppen ausgegrenzt. Damit einher geht das nationalstaatliche Bemühen der Homogenisierung von Kultur, die keineswegs die Voraussetzung des Nationalstaats ist, sondern sein, in vielen Bereichen nur unvollkommen erreichtes, Produkt. Für Frankreich hat etwa Eugene Weber (1976) detailliert nachgezeichnet, wie der Nationalstaat aus „Bauern“ „Franzosen“ gemacht hat - ein Prozess, der trotz massiver institutioneller Anstrengungen und Disziplinierungsmaßnahmen hundert Jahre dauerte. Die Unvollständigkeit kultureller Homogenisierung im Nationalstaat ließ sich in Deutschland plastisch an der Debatte über die ,deutsche Leitkultur" erkennen, die nicht zu einem Ergebnis kam, worin diese gemeinsame, „deutsche" Kultur denn eigentlich bestehe.

Die Konzepte der modemen Nation und der Pluralität der Kulturen haben sich parallel und in wechselseitiger Abhängigkeit entwickelt. Ein Konzept von Kultur, das Hybridität ins Zentrum stellt, kann nicht durch das nationalstaatliche Modell visualisiert werden, das etwa Landkarten mit verschiedenfarbigen, voneinander klar abgegrenzten Flächen zeichnet. Stattdessen müsste man sich vielleicht ein Bild im Stil des Impressionismus vorstellen, in dem zwar bestimmte Farbflecken und Konturen erkennbar sind, die aber gleichzeitig an den Rändern verlaufen, teils ineinander übergehen, oder Zwischenräume frei lassen. Anstelle von Differenz würde das Konzept Gemeinsamkeiten und Kontinuitäten betonen und zwar weniger im Sinne gemeinsamer Eigenschaften, als im Sinne von sozialen Prozessen und einer geteilten Fähigkeit zur Interaktion und Kommunikation,

5 Vgl. etwa die Kritik von Çağlar 1997. Für einen Überblick über die HybriditätsDebatte siehe Pieterse 2001 die alle möglichen „Elemente“ hervorbringen kann. So definiert etwa HansRudolf Wicker Kultur als „exclusively the ability to produce reciprocal symbolic relations and to form meaning through interaction" (1997: 39f.).

Die Idee der Hybridität ist jedoch bisher kaum aus dem akademischen Diskurs in den allgemein-gesellschaftlichen und politischen Diskurs eingesickert, wenn man einmal von Teilbereichen in Literatur, Kunst und Musik absieht. ${ }^{6}$ In der Debatte um Migration herrscht das konventionelle Verständnis von Kultur vor, wie die Diskussion um Leitkultur oder Multikultur zeigt. Die Debatte ist vom Paradigma der kulturellen Differenz geprägt. Darunter verstehe ich die $\mathrm{Zu}$ schreibung kultureller Differenz als Prämisse der Wahrnehmung und Auseinandersetzung. Migranten gelten a priori als Andere, als Fremde, unabhängig von der tatsächlichen Erfahrung. Die Zuschreibung als Prämisse kann die tatsächliche Erfahrung sogar weitgehend überdecken. So wird Einwanderern, auch der so genannten „dritten Generation" immer noch Fremdheit zugeschrieben. Diese Fremdheit basiert nicht darauf, dass man etwa einen Menschen türkischer Herkunft nicht kennt (dieselbe Fremdheit würde dann ja auch für „Eingeborene“ gelten, die ich nicht persönlich kenne), sondern darauf, dass man Einwanderern kollektiv und kategorisch essentielles Anderssein zuschreibt. ${ }^{7}$ Die Mechanik des Paradigmas lässt sich sehr gut an der seit einigen Jahren laufenden Debatte über das Deutschlernen nachvollziehen. Es ist gängige Praxis v.a. von Politikern geworden, Migranten dazu aufzufordern, Deutsch zu lernen. Als unbedarfter Zuhörer kann man aus der ständigen Widerholung der Forderung nur schließen, Migranten hätten bis heute generell nicht Deutsch gelernt. Das ist offensichtlich Unsinn. Damit will ich nicht bestreiten, dass es zahlreiche Migranten gibt, die nich hinreichend Deutsch sprechen und die Sprache dringend besser lemen sollten. Aber die pauschale Aufforderung „Lernen Sie Deutsch!" an „die Migranten“, die etwa der ehemalige Bundespräsident Rau in seiner vielzitierten „Berliner Rede“ im Jahr 2000 geäußert hat (Rau 2000), sagt mehr über die Wahmehmung und diskursive Konstruktion von "Migranten" in Deutschland aus als über die tatsächlichen Sprachkenntnisse eben dieser Migranten. Die pauschale Forderung kennzeichnet „Migranten“ wiederum generell als defizitär und anders und grenzt sie damit aus.

Das Paradigma kultureller Differenz, das eindeutige Unterschiede als gegeben voraussetzt und sie damit im gesellschaftlichen Diskurs gleichzeitig reproduziert, dominiert die deutsche Politik gegenüber Migranten und Migration. Das Problem der Einwanderung ist, dass die Eingewanderten anders sind als „wir“, sie sprechen nicht „unsere“ Sprache, haben andere Werte und Vorstellungen, andere Praktiken. Über dieses Grundmuster der Wahrnehmung kann nicht hinwegtäuschen, dass sich die Verortung der Differenz mit der Zeit verschiebt, dass

6 Zu HipHop und Hybridität siehe El-Tayeb 2004

7 Zum Paradigma kultureller Differenz in Bezug auf Einwanderer aus der Türkei siehe Sökefeld 2004. 
etwa heute andere Gruppen als die paradigmatischen Fälle des Anderen gelten als in der Vergangenheit, oder dass die Differenz mit anderen Mitteln konstruiert wird. So wird Differenz heute in erster Linie als religiöse Differenz betrachtet, während vor nicht allzu langer Zeit Differenz v.a. in nationalen Kategorien gedacht wurde. Waren bis vor einigen Jahren v.a. „die Türken“ die anderen, so sind es heute „die Muslime“.

Nach wie vor werden gesellschaftliche Konflikte durch kulturelle Unterschiede erklärt; allerdings werden Einwanderer in ihrem Anderssein nicht mehr, wie zu Zeiten der „Differenzhypothese“, durch den Verweis auf ihre andere Kultur ,entschuldigt“. In dieser Hinsicht ist das Ende der Toleranz gekommen. Anpassung, Assimilation wird gefordert.

Die unhinterfragte Verknüpfung von Migration mit dem Paradigma kultureller Differenz und von Differenz mit Konflikt geht implizit von der Prämisse aus, dass erstens die Gesellschaft ohne Einwanderung kulturell homogen wäre und dass zweitens eine solche homogene Gesellschaft, so es sie denn gäbe, konfliktfrei wäre. Beide Annahmen sind offensichtlich unhaltbar, und es ist äußerst fraglich, ob die Politik der Assimilation und Homogenisierung, falls sie denn uberhaupt erfolgreich sein könnte, tatsächlich zur Verringerung von Konflikten führen würde.

Recht sicher lässt sich aber das Gegenteil dieser Vorstellung begründen, dass sich nämlich die diskursive Betonung der Differenz, die mit Misstrauen verknüpfte Prämisse des Andersseins der Migranten, welche die unvermeidliche Ruickseite der Forderung nach Assimilation ist, in gesellschaftlichen Strukturen und Praktiken der Ausgrenzung niederschlägt, und zwar von beiden Seiten. Der gesellschaftliche Deutungs- und Handlungsspielraum wird dadurch eingeschränkt. Integration, egal, ob man darunter nur ein anderes Wort für Anpassung versteht oder das Ziel gleichberechtigter gesellschaftlicher Teilhabe, wird erschwert und letztlich vielleicht sogar unmöglich gemacht.

\section{Islam als das Paradigma des Anderen}

Ich möchte diesen fatalen Zusammenhang am Beispiel des Verhältnisses zu Muslimen illustrieren. „Der Islam" - selbst ein diskursiv homogenisierte Konstrukt, das die tatsächliche Vielfalt muslimischer Lebensstile negiert - gilt heute als das radikal Andere der deutschen oder insgesamt „westlichen“ Kultur. Trotz aller Aufrufe zu Dialog und Verständigung wird die Differenz zwischen islamischer und deutscher Kultur als weitgehend unüberbrückbar betrachtet. Der Islam gilt als „fremde" Religion. Er wird ganz überwiegend mit Negativem in Verbindung gebracht. Die Assoziation des Islam mit Gefahr und Gewalt ist so gängig geworden, dass beispielsweise der „Spiegel“ kommentarlos einen Artikel über Krimi- nalität und innere Sicherheit in Deutschland mit einem Foto betender Muslime illustrieren kann. ${ }^{8}$

Nehmen wir das Beispiel des Kopftuchstreits und der Lehrerin Fereshta Ludin. Die dominante, politisch durchgesetzte Interpretation des Falles ist ebenso bekannt wie seine Konsequenz: In den vielen Bundesländern dürfen muslimische Lehrerinnen kein Kopftuch tragen. Das Kopftuch gilt als Symbol für Zwang und/oder Islamismus und wird als Ausweis der radikalen Andersheit derjenigen verstanden, die es tragen oder befürworten.

Das Kopftuch muss aus dem staatlichen Raum - als solcher wird die Schule verstanden - ausgeschlossen werden, da sonst die Subversion des Eigenen droht, die Subversion der Neutralität des Staates (der sich der Religion gegenüber jedoch alles andere als neutral verhält), oder ganz generell die der eigenen Lebensweise, der eigenen Normen. Soweit, etwas überspitzt dargestellt, die vorherrschende Interpretation des Kopftuchstreits.

Man hätte den Fall Fereshta Ludins auch völlig anders verstehen und behandeln können: Eine junge Muslimin will Lehrerin an einer deutschen Schule werden. Sie will sich offensichtlich integrieren. Man hätte die erste kopftuchtragende muslimische Lehrerin an einer Schule Baden-Württembergs gar als ein gelungenes Beispiel für Integration feiern können. Auch die Tatsache, dass Fereshta Ludin sich des Rechtssystems bediente, um ihr Interesse und das, was sie als ihr Recht empfand, durchzusetzen, hätte man als positives Beispiel der Integration einer Migrantin im Sinne gesellschaftlicher Partizipation verstehen können.

Warum sind diese Interpretationen der Geschichte im deutschen Kontext unmöglich und würden vermutlich bestenfalls als "naiv" tituliert? Der Grund ist nicht das Kopftuch „an sich“. In Großbritannien zum Beispiel ist eine muslimische Lehrerin mit Kopftuch ebenso wenig ein prinzipielles Problem wie ein turbantragender Sikh im Polizeidienst. Der Grund liegt darin, dass in Deutschland das Kopftuch als Symbol der Differenz schlechthin wahrgenommen wird. Kernpunkt der Argumentation der Kopftuchgegner war das Urteil, dass das Kopftuch nicht nur ein religiöses Symbol ist. Diese Wahrnehmung ist eine Prämisse der Debatte, die etwa durch die Beteuerungen von Kopftuchträgerinnen, dass sie das Tuch ohne Zwang tragen und dass sie damit keine politische Absicht verbinden, nicht widerlegt werden kann. Da das Thema so heikel ist und ich jedes Missverständnis ausschließen möchte, will ich hier betonen, dass ich damit nicht sage, dass ,das Kopftuch" nicht ein Instrument des Zwangs und der Unterdrückung von Frauen oder mit politischen Botschaften verknüpft sein kann. Aber es muss nicht in jedem Fall so sein. Yasemin Karakaşoğlu (2003) argumentierte in ihrem Gutachten für das Bundesverfassungsgericht im Kopftuchstreit mit der Pluralität der Motive von Musliminnen, das Kopftuch zu tragen, und plädierte daher für eine Einzelfallprüfung statt für die generelle Ausschließung des Kopftuchs. Diese Auffassung wird auch von anderen sozialwissenschaftlichen Studien

8 Der Spiegel Nr. 25, 2005, S. 31. 
über Musliminnen in Deutschland gestützt (vgl. Nökel 2002; Klinkhammer 2000). Diese differenzierte Perspektive hat in der öffentlichen Diskussion in Deutschland jedoch kaum eine Chance. Hier dominierte die essentialistische Sichtweise, die das Kopftuch an sich unter Unterdrückungs- und Islamismusverdacht stellte, und für die es folglich nur zwei Gründe geben kann, das Tuch zu tragen: Entweder frau wird dazu gezwungen, oder sie ist eine Islamistin. Das Kopftuch wird so zu einem zentralen Punkt der Abgrenzung zwischen „uns“ (aufgeklärt, modern, fortschrittlich, säkular) und den „Anderen“ (rückständig und gefährlich, von der Religion dominiert). Die ausgrenzende, verallgemeinernde Zuschreibung der Differenz, die sich auch von gegenteiligen Forschungsergebnissen nicht erschüttern lässt, ist die Prämisse des dominanten Diskurses. Daraus entsteht eine wechselseitige Polarisierung zwischen „Deutschen" und „muslimischen Migranten" (schon die Abgrenzung ist falsch, es gibt ja genügend muslimische Deutsche), die der wechselseitigen Verständigung sicher nicht förderlich ist.

Charakteristisch ist auch, dass die Tatsache, dass Fereshta Ludin Deutsche ist, nicht dazu führt, die Konzeption des Eigenen (des Deutschen) zu überdenken, die Differenz in das Eigene mit hineinzunehmen und das Eigene „plural“" zu denken. Im Gegenteil, der umgekehrte Schluss wird gezogen: In einem solchen Fal kann Einbürgerung eigentlich nur „falsch" oder gar missbräuchlich sein. Und so werden Prüfungsprozeduren entwickelt, die im Einbürgerungsverfahren garantieren sollen, künftig die Falschen zu identifizieren und auszuschließen. In Deutschland kann man jedoch, trotz der Reform des Staatsangehörigkeitsrechts, den Eindruck bekommen, als sei eigentlich jede Einbürgerung „falsch“. Denn die Einbürgerung macht aus Ausländern nicht wirklich Deutsche, sie macht aus ihnen nur Ausländer mit einem deutschen Pass.

Das zeigt die Erfahrung zahlreicher eingebürgerter Deutscher, die im Alltag in fast jeder Hinsicht nach wie vor als Ausländer wahrgenommen und behandelt werden. Untersuchungen zeigen, dass diese Erfahrung der Eingebürgerten inzwischen zu einem Hinderungsgrund für weitere Einbürgerungen wird, denn die Erfahrung sagt: Es ändert sich ja doch nichts, warum soll man sich dann einbürgern lassen (vgl. Sauer 2001)? Diese Erfahrung gilt bei weitem nicht nur für kopftuchtragende oder bärtige Islamisten, sondern auch für Personen, die nach landläufiger Vorstellung eigentlich gut integriert sein müssten. Dazu ein Ausschnitt aus einem Interview des „Spiegel“ mit Bassam Tibi, der sich in einem Gespräch über Islamismus selbst als Beispiel für ,gescheiterte Integration“ bezeichnet, obwoh er doch als Vorkämpfer eines aufgeklärten und moderaten „Euro-Islam“ gefeiert wird:

Der Spiegel: „Erstaunlich ist aber doch, dass Sie sich selbst auch als ein Beispiel gescheiterter Integration sehen. Sie lehren seit über 30 Jahren an einer deutschen Universität, Sie haben 26 Bücher auf Deutsch geschrieben und das Bundesverdienstkreuz haben Sie auch. Warum um alles in der Welt sind Sie nicht integriert?"
Tibi: „Es geht um ein Gefühl der Zugehörigkeit. In Deutschland ist es kein Widerspruch zu sagen, Herr Tibi ist Syrer und hat einen deutschen Pass. In Frankreich ist es das aber, und in Amerika ist es ein Grund für eine Klage, weil man jemanden von der amerikanischen Gesellschaft ausschließt. Deutscher, das bin ich auch nach 40 Jahren hier noch nicht. ${ }^{: 99}$

Das Hindernis für die Integration besteht nicht darin, dass Tibi nicht genug für seine Integration geleistet hätte. Das Hindernis besteht in der dominanten Vorstellung davon, was eigentlich „deutsch" ist. Sie ist die andere Seite des Paradigmas kultureller Differenz: Was nach landläufiger Vorstellung „fremd“ und anders ist, kann nicht deutsch sein. Das Eigene wird durch den Ausschluss des Anderen definiert. Nach wie vor kann man nicht Deutscher werden. Man muss als Deutscher geboren sein. Ohne eine Änderung der gängigen Vorstellung davon, was „deutsch" ist, kann Integration nicht wirklich gelingen.

In einem Beitrag über „Citizenship of Muslims in Germany" vergleicht Werner Schiffauer die „Zivilkultur" Deutschlands in Bezug auf Einwanderung und Einbürgerung mit der anderer europäischer Staaten. Während beispielsweise in den Niederlanden Einbürgerung als Anfang eines Prozesses der Integration betrachtet wird, gilt sie in Deutschland als Abschluss der Integration, die dann, wie das Zitat von Tibi zeigt, doch nicht gelingt. Man wird sozusagen für die Integration, die in diesem Zusammenhang weitgehend als Assimilation zu verstehen ist, mit der Einbürgerung belohnt. Damit ist in Deutschland die Auffassung verknüpft, Integration setze voraus, dass man sich mit einem common good und der politischen Gemeinschaft insgesamt identifiziere. Während in Deutschland Integration also als die Identifikation mit einer Art Substanz (ich würde sagen „Kultur") impliziert, kommt es in England oder Frankreich darauf an, dass man sich mit Regeln des Zusammenlebens einverstanden erklärt. Das common good entsteht dann aus der Beachtung der Regeln. Das Vertrauen in Regeln ist in Deutschland jedoch gering ausgeprägt. Es überwiegen das Misstrauen und der Verdacht, Regeln und Verfahren könnten manipuliert und missbraucht werden (vgl. Schiffauer 2006). Daher die Verschärfung der Einbürgerungsprüfungen, daher die Wiederausbürgerung von zahlreichen ehemaligen türkischen Staatsbürgern, die nach der Einbürgerung die türkische Staatsbürgerschaft wieder angenommen hatten und damit gegen das Verbot der doppelten Staatsbürgerschaft verstießen. ${ }^{10}$

9 Der Spiegel, Nr. 40, 2. Oktober 2006, S. 49.

$10 \mathrm{Im}$ letzten Jahr wurde verschiedentlich geäußert, dass bis zu 50.000 Eingebürgerte die Ausbürgerung zu befürchten hätten. Leider sind mir keine nachprüfbaren Zahlen bekannt, wie viele insgesamt tatsächlich die deutsche Staatsbürgerschaft wieder verloren haben. Einem Bericht der tageszeitung zufolge wurden jedoch allein in Bremen bis September 2005 aus 520 Deutschen wieder Türken (taz Bremen, 3. September 2005). 


\section{Das Paradigma kultureller Differenz: Methodische Konsequenzen für die Sozialwissenschaften}

Ich habe für diesen Beitrag das Paradigma kultureller Differenz definiert als die Zuschreibung kultureller Differenz als Prämisse der Wahrnehmung und Auseinandersetzung in der Debatte über Migration in Deutschland. Das Paradigma kultureller Differenz definiert das Eigene und das Andere und führt dazu, dass Migranten als eigenständige soziale Kategorie betrachtet werden. Diese soziale Kategorie gilt als problembeladen, nicht nur weil „Fremdheit“ ohnehin als Problem betrachtet wird, sondern weil sie mit allen möglichen sozialen Problemen, heute v.a. im Bildungsbereich, verknüpft wird. Ich betrachte die Konstruktion dieser Kategorie selbst als Problem, weil sie eine Dynamik wechselseitiger Abgrenzung in Gang setzt und somit als self-fulfilling prophecy tatsächlich den gesellschaftlichen Zusammenhalt gefährden kann.

In der Vergangenheit hat die wissenschaftliche Beschäftigung mit Migration und ihren Folgephänomenen häufig zur Ausgrenzung von Migranten und PostMigranten als Sonder- und Problemkategorie beigetragen.

Andreas Wimmer und Nina Glick Schiller haben in viel beachteten Beiträgen den methodologischen Nationalismus der Sozialwissenschaften, besonders in der Untersuchung von Migrationsphänomenen, aufgezeigt und kritisiert. Sie verstehen unter methodologischem Nationalismus die „Naturalisierung des Nationalstaates durch die Sozialwissenschaften“" (Wimmer/Glick Schiller 2003: 578; vgl. Wimmer/Glick Schiller 2002). Zentrales Element des methodologischen Nationalismus ist die Annahme, dass eine Gesellschaft im Nationalstaat ein "Container" ist, der eine Kultur, ein politisches System, eine Wirtschaft und eine abgegrenzte soziale Gruppe beinhaltet. Das ist eine Annahme, der in Deutschland das Paradigma kultureller Differenz entspricht. Erst unter der Prämisse des methodologischen Nationalismus wird Migration zu einem problematischen Sonderphänomen, das mit speziellen Instrumenten und Programmen behandelt werden muss. Wimmer und Glick Schiller verweisen zum Beispiel auf Statistiken, in denen Migranten etwa im Hinblick auf Bildungserfolg oder Arbeitslosigkeit mit dem nationalen Durchschnitt verglichen werden, dabei schlecht abschneiden, und somit als Problemgruppe identifiziert werden. Das Problem besteht aber gerade darin, dass Migranten als Gruppe ausgesondert und mit nationalen Mittelwerten verglichen werden, anstatt sie zu den Segmenten der Gesamtbevölkerung in Beziehung zu setzen, die ihnen nach sozialen Indikatoren wie Einkommen oder Bildungsschichtzugehörigkeit ähneln (vgl. Wimmer/Glick Schiller 2003: 584). Sabine Mannitz (2006: 9) spricht hier von der beobachtungsstrukturellen Diskriminierung von Einwanderern und ihren Nachkommen.

Ebenso entsteht ein „problematisches" Bild von Migranten dadurch, dass v.a. Problemphänomene etwa in urbanen Ballungsräumen untersucht werden, die ohnehin unter sozialen Problemen leiden, während eher ,unproblematische“ Regionen außerhalb der Betrachtung bleiben. Ich glaube, dass etwa die Erforschung von Migrationsfolgephänomenen in schwäbischen Kleinstädten zu anderen Ergebnissen führen würde, als die üblichen Studien über Berlin Kreuzberg, Duisburg Marxloh oder Hamburg Wilhelmsburg. Sie finden nur viel zu wenig statt, weil das „Unproblematische“ kaum als untersuchungswürdig gilt. Mir geht es hier nicht darum, das Bild einer heilen Welt zu zeichnen, sondern darum, der Überproblematisierung von Migration und ihren Folgen entgegenzuwirken. So möchte ich zum Abschluss meines Vortrags drei Vorschläge machen, wie die Sozialwissenschaften dem Paradigma kultureller Differenz und der Überproblematisierung von Migration und „Migranten“ bzw. ihren Nachkommen entgegenwirken können:

(1) Die Sozialwissenschaften sollten verstärkt betonen, dass Migration ein „,normales" Phänomen ist, das erst im Rahmen des Nationalstaats mit seiner Fiktion kultureller Homogenität der Nation und seinen Bemühungen um Ausschließung der anderen zum Ausnahmefall und „Problem" wird. Das heißt, wir müssen den methodologischen Nationalismus, mit dem wir uns die Perspektive des Nationalstaats zu Eigen machen, ablegen. Dazu gehört, zu betonen, dass auch eine Gesellschaft ohne Migration weder kulturell homogen noch konfliktfrei wäre.

(2) Wir sollten sehr bewusst und reflektiert mit den Begriffen „Migration“ und „Migranten" u.Ä. umgehen. Diese Begriffe sollten auf die Menschen beschränkt bleiben, die tatsächlich gewandert sind. Im akademischen Rahmen etwa von „Migranten der dritten Generation“" zu reden, bedeutet, sich dem ausschließenden allgemein-gesellschaftlichen Diskurs anzuschließen. Die Rede von „Menschen mit Migrationshintergrund“ finde ich ebenfalls nicht besonders geeignet, weil sehr bemüht. In der englischsprachigen Literatur ist zum Teil von post-migrants und post migration die Rede. Ich kann leider keine idealen begrifflichen Alternativen anbieten, aber ich denke, wir sollten uns ernsthaft auf die Suche danach begeben.

(3) Die Forschung sollte Migration nicht von vornherein als ein Problem betrachten, sondern auch die Normalität von Migration untersuchen. Forschungen jenseits städtischer Problemgebiete sollten durchgeführt werden. Dabei sollten Migranten und Post-Migranten auch nicht von vornherein als eigenständige Kategorie betrachtet werden. $\mathrm{Ob}$ sie das sind oder nicht, das sollte eine empirische Frage sein. Es käme darauf an, etwa Interaktionsräume zu beobachten und zu untersuchen, ob es zum Beispiel nicht viel mehr Beziehungen jenseits herkömmlicher Kategorien und angenommener Grenzen gibt, als gemeinhin vermutet.

Wenn das Paradigma kultureller Differenz dazu fuihrt, dass Einwanderern und ihren Nachkommen die Anerkennung als ,normale“ Mitglieder der Gesellschaft 
verweigert wird, dann haben wir damit auf die Dauer ein massives politisches Problem. Die Sozialwissenschaften sollten verstärkt bemüht sein, diese Ausgrenzung nicht zu reproduzieren oder zu verstärken, sondern sie in Frage zu stellen und über sie hinaus zu denken.

\section{Literatur}

Çağlar, Ayşe (1997): Hyphenated Identities and the Limits of ,Culture'. In: Tariq Modood/Pnina Werbner (Hg.), The Politics of Multiculturalism in the New Europe, London: Zed Books, S. 169-185.

El-Tayeb, Fatima (2004): Kanak Attak! HipHop und (Anti-)Identitätsmodelle der „Zweiten Generation“. In: Martin Sökefeld (Hg.), Jenseits des Paradigmas kultureller Differenz: Neue Perspektiven auf Einwanderer aus der Türkei, Bielefeld: transcript, S. 95-110.

Fuchs, Martin/Berg, Eberhard (1993): Phänomenologie der Differenz. Reflexionsstufen ethnographischer Repräsentation. In: Eberhard Berg/Martin Fuchs (Hg.), Kultur, soziale Praxis, Text. Die Krise der ethnographischen Repräsentation, Frankfurt/M.: Suhrkamp, S. 11-108.

Giddens, Anthony (1984): Interpretative Soziologie: Eine kritische Einführung, Frankfurt/M.: Campus.

Griese, Hartmut (2002): Einleitung: Was ist eigentlich das Problem am „Ausländerproblem"? In: Hartmut Griese/Elçin Kürşat-Ahlers/Rainer Schulte/Massoud Vahedi (Hg.), Was ist eigentlich das Problem am "Ausländerproblem"?, Frankfurt/M.: IKO-Verlag, S. 25-45.

Gontovos, Konstantinos (2000): Psychologie der Migration. Über die Bewältigung von Migration in der Nationalgesellschaft, Hamburg: Argument.

Hamburger, Franz/Sens, Lydia/Walter, Otto (1984): Über die Unmöglichkeit, Politik durch Pädagogik zu ersetzen. Reflexionen nach einer Untersuchung ,Bedingungen und Verfestigungsprozesse der Delinquenz bei ausländischen Jugendlichen'. In: Hartmut Griese (Hg.), Bilanz und Kritik der Gastarbeiterforschung und Ausländerpädagogik, Opladen: Leske + Budrich, S. 32-42.

Karakaşoğlu, Ayse (2003): Stellungnahme zu den Motiven von jungen Musliminnen in Deutschland für das Anlegen eines Kopftuches, http://www.wzberlin.de/zkd/aki/files/karakasoglu_gutachten_kopftuch.pdf, 10.10 .06

Klinkhammer, Grit (2000): Moderne Formen islamischer Lebensführung. Eine qualitativ-empirische Untersuchung zur Religiosität sunnitisch geprägter Türkinnen der zweiten Generation in Deutschland, Marburg: Diagonal.

Mannitz, Sabine (2006): Die verkannte Integration. Eine Langzeitstudie unter Heranwachsenden aus Immigrantenfamilien, Bielefeld: transcript.

Nökel, Sigrid (2002): Die Töchter der Gastarbeiter und der Islam. Zur Soziologie alltagsweltlicher Annerkennungspolitiken, Bielefeld: transcript.
Pieterse, Jan Nederveen (2001): „Hybridity - so what? The anti-hybridity backlash and the riddles of recognition". Theory, Culture and Society 18, S. 219245.

Rau, Johannes (2000): Ohne Angst und ohne Träumereien: Gemeinsam in Deutschland leben. Berliner Rede von Bundespräsident Johannes Rau im Haus der Kulturen am 12. Mai 2000, http://www.bundespraesident.de/Redenund-Interviews/Berliner-Reden, 12090/Berliner-Rede-2000.htm, 10.10.2006

Sauer, Martina (2001): Die Einbürgerung türkischer Migranten in Deutschland. Befragung zu Einbürgerungsabsichten und dem Für und Wider der Einbürgerung. In: Andreas Goldberg/Dirk Halm (Hg.), Migrationsbericht des Zentrums für Türkeistudien 2002, Münster: Lit, S. 165-228.

Schiffauer, Werner (2006): Enemies within the gates: the debate about the citizenship of Muslims in Germany. In: Tariq Modood/Anna Triandafyllidou/ Ricard Zapata-Barrero (Hg.), Multiculturalism, Muslims and citizenship: A European approach, London: Routledge, S. 94-116.

Schrader, Achim/Nikles, Bruno/Griese, Hartmut (1976): Die zweite Generation. Sozialisation und Akkulturation ausländischer Kinder in der Bundesrepublik, Kronberg: Athenäum.

Sökefeld, Martin (2001): Der Kulturbegriff in der Ethnologie und im öffentlichen Diskurs - eine paradoxe Entwicklung? In: Georg Stöber (Hg.), „Fremde Kulturen " im Geographieunterricht. Analysen, Konzeptionen, Erfahrungen, Hannover: Verlag Hahnsche Buchhandlung, S. 119-137.

Sökefeld, Martin (2004): Das Paradigma kultureller Differenz: Zur Forschung und Diskussion über Migranten aus der Türkei in Deutschland. In: Ders. (Hg.), Jenseits des Paradigmas kultureller Differenz: Neue Perspektiven auf Einwanderer aus der Türkei in Deutschland, Bielefeld: transcript, S. 9-33.

Stolcke, Verena (1995): „Talking culture: New boundaries, new rhetorics of exclusion in Europe". Current Anthropology 36, S. 1-13.

Treibel, Annette (1988): Engagement und Distanzierung in der westdeutschen Ausländerforschung. Eine Untersuchung ihrer soziologischen Beiträge, Stuttgart: Ferdinand Enke Verlag.

Weber, Eugene (1976): Peasants into Frenchmen: The modernization of rural France 1870-1914, Stanford: Stanford University Press.

Wicker, Hans-Rudolf (1997): From complex culture to cultural complexity. In: Pnina Werbner/Tariq Modood (Hg.), Debating cultural hybridity: Multicultural identities and the politics of anti-racism, London: Zed, S. 29-45.

Wimmer, Andreas/Glick Schiller, Nina (2002): Methodological nationalism and beyond: nation-state building, migration and the social sciences. Global Networks 2, S. 301-334.

Wimmer, Andreas; Glick Schiller, Nina (2003): Methodological nationalism, the social sciences, and the study of migration: An Essay in Historical Epistemology. International Migration Review 37, S. 576-610. 